\title{
Research Article \\ Exponential Stabilizability of Switched Systems with Polytopic Uncertainties
}

\author{
Xia Zhang, ${ }^{1}{ }^{2}$ Zun-Quan Xia, ${ }^{1}$ and Yan Gao ${ }^{3}$ \\ ${ }^{1}$ School of Mathematical Sciences, Dalian University of Technology, Dalian 116024, China \\ ${ }^{2}$ School of Information, Zhejiang University of Finance and Economics Dongfang College, \\ Haining 314408, China \\ ${ }^{3}$ School of Management, University of Shanghai for Science and Technology, \\ Shanghai 200093, China
}

Correspondence should be addressed to Xia Zhang, sdzhx2002@163.com

Received 5 October 2012; Revised 8 December 2012; Accepted 10 December 2012

Academic Editor: Piyapong Niamsup

Copyright (C 2012 Xia Zhang et al. This is an open access article distributed under the Creative Commons Attribution License, which permits unrestricted use, distribution, and reproduction in any medium, provided the original work is properly cited.

The exponential stabilizability of switched nonlinear systems with polytopic uncertainties is explored by employing the methods of nonsmooth analysis and the minimum quadratic Lyapunov function. The switchings among subsystems are dependent on the directional derivative along the vertex directions of subsystems. In particular, a sufficient condition for exponential stabilizability of the switched nonlinear systems is established considering the sliding modes and the directional derivatives along sliding modes. Furthermore, the matrix conditions of exponential stabilizability are derived for the case of switched linear system and the numerical example is given to show the validity of the synthesis results.

\section{Introduction}

In the last two decades, there has been increasing research in stability analysis and control design for switched systems and many results have been studied on stability and stabilizability problems for various types of switched systems [1-9]. Many interesting results for different kinds of problems of switched systems can be found in some books [10,11]. The motivation of studying the switched system is out of the fact that many practical systems are inherently multimodal, and several dynamical subsystems are required to describe their behavior which may depend on various environmental factors. Sometimes there are some systems that cannot be asymptotically stabilized by a single continuous feedback control rule but can be stabilized by switching rule [3]. 
It is very important to investigate switched systems which contain uncertainties due to modelling errors, aging disturbance, and complex environment in realistic problems. One important type of uncertain systems are switched systems which are composed of polytopic uncertainty subsystems. As pointed out in [12], polytopic uncertainties exist in many real systems, and most of the uncertain control systems can be approximated by systems with polytopic uncertainties. The polytopic uncertain systems are less conservative than systems with norm bounded uncertainties [13]. Recently, the stability and stabilization problems for both continuous-time and discrete-time switched systems with polytopic uncertainties are investigated in [14, 15]. In particular, the paper [14] investigated the quadratic stabilizability problem via state feedback, and provided sufficient conditions to be quadratically stabilized for the switched systems which being composed of two subsystems. More recently, necessary and sufficient conditions for continuous-time case via state feedback are proved in the paper $[16,17]$.

Lyapunov theory is a very important approach to stability analysis or stabilization for switched systems, and the construction of Lyapunov functions is one of the fundamental problem in system theory. The most popular types of Lyapunov function are quadratic functions, piecewise-linear functions, and piecewise affine functions $[9,18-20]$. Furthermore, there are some results for the systems with time-delay [21, 22]. Paper [22] investigates the stability and stabilization properties of linear switched time delay dynamic systems subject to; in general, multiple uncommensurate known internal point delays based on Lyapunovs stability analysis via appropriate Krasovsky-Lyapunovs functionals and the related stability study is performed to obtain both delay independent and delay dependent results. In [9], the authors studied the exponential stabilization problem for discrete-time switched linear systems based on a special control Lyapunov function which can make the hybrid-control policy of the related switched system be derived analytically and computed efficiently. Hu and Lin [23] proposed a composed quadratic Lyapunov function for constrained control systems. The composite quadratic Lyapunov function turned out to be very effective in dealing with some constrained control systems as well as a class of more general nonlinear systems [4, 24-26].

Motivated by the methods in [4], we consider the exponential stabilizability problems of switched nonlinear systems with polytopic uncertain subsystems using the composite quadratic functions. We mainly use the minimum quadratic function. The contributions in our work is that, the analysis of possible sliding modes in the switched systems and employing the nonquadratic Lyapunov functions to reduce the conservatism in references $[14,16,17]$. We extend the main result of [14] and establish the matrix conditions of exponential stabilizability for the switched linear systems.

The remainder of this paper is organized as following. In Section 2, we briefly review some conceptions especially the switched systems with polytopic uncertainties and the minimum quadratic functions. Then, in Section 3, the exponential stabilizability results based on the minimum functions are established, and the conditions as matrix inequalities are derived for the switched linear system. Moreover an example is given to demonstrate the effectiveness of our method. Finally, concluding remarks are given in Section 4 and some definitions or conclusions from nonsmooth analysis are listed in the appendix.

Notations. We use $I\left[k_{1}, k_{2}\right]$ to represent the set of integers $\left\{k_{1}, k_{1}+1, k_{1}+\right.$ $\left.2, \ldots, k_{2}\right\} ; \nabla V(x)$ denotes the gradient of $V$ at $x$ and $\partial V(x)$ the subdifferential of $V$ at $x$; $\dot{V}(x ; \zeta)$ stands for the one-sided directional derivative of $V$ at $x$ along $\zeta ; \operatorname{co}\{S\}$ denotes the convex hull of a set $S$. 


\section{Switched Systems and the Minimum Quadratic Function}

Switched system is a hybrid dynamical system composed by a family of continuous-time or discrete-time subsystems with a rule orchestrating the switching between the subsystems $[1,2]$. In this paper, we consider the time-invariant switched nonlinear systems

$$
\dot{x}(t)=f_{\sigma(x, t)}(x(t)),
$$

where $x(t) \in R^{n}$ is the state vector and $\sigma(x, t)$ is the switching rule defined by $\sigma(x, t)$ : $R^{n} \times R^{+} \rightarrow I[1, N], R^{+}$denotes nonnegative real numbers. Therefore, the switched system is composed of $N$ continuous time subsystems which are expressed as

$$
\dot{x}(t)=f_{i}(x(t)), \quad i \in I[1, N] .
$$

We assume that all subsystems are uncertain systems of polytopic type described as

$$
f_{i}=\sum_{j=1}^{N_{i}} \lambda_{i j} f_{i j}, \quad i \in I[1, N]
$$

where $f_{i j}: R^{n} \rightarrow R^{n}, j \in I\left[1, N_{i}\right]$ are known and in locally Lipschitzian. They are called vertex directions of the subsystems. $N_{i}, i \in I[1, N]$ are integers. $\lambda_{i j} \geq 0, j \in I\left[1, N_{i}\right]$ are polytopic uncertain parameters for $i \in I[1, N]$ and satisfy $\sum_{j=1}^{N_{i}} \lambda_{i j}=1$.

Define the minimum function

$$
V_{\min }(x)=\min \left\{V_{j}(x) \mid j \in I[1, J]\right\}
$$

where $\left\{V_{1}, V_{2}, \ldots, V_{J}\right\}$ is a set of differentiable, positive definite, and radially unbounded functions, which are zero at $x=0$.

The function $V_{\min }(x)$ is positive definite and homogeneous of degree two [23]. It is established from the theory of nonsmooth analysis that the function is not convex and not differentiable everywhere even if the functions $V_{i}(x)$ are all differentiable.

For a vector field $\dot{x}=h(x)$, we have $V(x(t+\Delta t)) \approx V(x+h(x) \Delta t)$ for $\Delta t>0$ small enough. Thus directional derivative $\dot{V}(x ; h(x))$ measures the time derivative of $V$ at $x$ along the trajectory. For this reason the exponential stabilizability of the switched systems in our paper can utilize the directional derivative of the minimum quadratic function.

\section{Exponential Stabilizability of the Switched Systems}

There are many methods to construct switching rule for the stabilizability of switched systems [4, 14, 27]. Just as the paper [4], our switching rule is constructed by employing the minimum function and its directional derivative. We select the minimum function (2.4), which is constructed from $J$ general functions $V_{j}(x)$, where each $V_{j}(x)$ is continuously differentiable.

Now we consider the stability of a closed loop system $\dot{x}=f_{\sigma(x, t)}(x)$ by examining the directional derivative of $V_{\min }$ along its trajectories. 
Proposition 3.1. Consider the closed loop systems (2.1). Construct the switching rule as follows:

$$
\sigma(x)=\underset{i \in I[1, N]}{\arg \min }\left\{\max _{j \in I\left[1, N_{i}\right]} \dot{V}_{\min }\left(x ; f_{i j}(x)\right)\right\}
$$

Define

$$
\mu(x):=\min _{i \in I[1, N]}\left\{\max _{j \in I\left[1, N_{i}\right]} \dot{V}_{\min }\left(x ; f_{i j}(x)\right)\right\}
$$

When $x$ is not in a sliding mode, $\dot{V}_{\min }(x ; \dot{x})=\dot{V}_{\min }\left(x ; f_{\sigma(x)}(x)\right) \leq \mu(x)$.

Proof. When $x$ is not in a sliding mode, then $\dot{x}$ may equal to a $f_{i}(x) . i$ is chosen by the switching rule $\sigma(x)$. Then

$$
\begin{aligned}
\dot{V}_{\min }(x ; \dot{x}) & =\dot{V}_{\min }\left(x ; \sum_{j=1}^{N_{i}} \lambda_{i j} f_{i j}(x)\right) \\
& =\sum_{j=1}^{N_{i}} \lambda_{i j} \dot{V}_{\min }\left(x ; f_{i j}(x)\right) \\
& \leq \max _{j \in I\left[1, N_{i}\right]}\left\{\dot{V}_{\min }\left(x ; f_{i j}(x)\right)\right\} \\
& =\min _{i \in I[1, N]}\left\{\max _{j \in I\left[1, N_{i}\right]}\left\{\dot{V}_{\min }\left(x ; f_{i j}(x)\right)\right\}\right\} \\
& =\mu(x) .
\end{aligned}
$$

The proof is completed.

From a practical point of view, a sliding mode is very important and it is often unavoidable in the switched systems. So we need to pay particular attention to sliding modes. When there is a sliding mode, we assume that $I_{\mathrm{sm}}$ be the set of indices of subsystems involved in the sliding mode. Then $\dot{x}$ may not equal to any of $f_{i}(x)$, instead $x(t)$ stay on the switching surface and $\dot{x}$ is a convex combination of those $f_{i}(x)^{\prime} \mathrm{s}$, that is,

$$
\dot{x}=\sum_{i \in I_{\mathrm{sm}}} \alpha_{i} f_{i}(x), \quad i \in I_{\mathrm{sm}}
$$

where $\alpha_{i} \geq 0, \sum_{i \in I_{\text {sm }}} \alpha_{i}=1$ (see [28]).

Proposition 3.2. Consider the closed loop systems (2.1) with the switching rule (3.1). Assuming a sliding mode involving subsystems $\dot{x}=f_{i}(x), i \in I_{\mathrm{sm}}$, then for each $x_{0}$ in this sliding mode,

$$
\dot{V}_{\min }\left(x_{0} ; f_{i}\left(x_{0}\right)\right) \leq \mu\left(x_{0}\right), \quad \forall i \in I_{\mathrm{sm}} .
$$


Moreover, along the sliding direction $\sum_{i \in I_{\mathrm{sm}}} \alpha_{i} f_{i}\left(x_{0}\right)$, one has

$$
\dot{V}_{\min }\left(x_{0} ; \sum_{i \in I_{\mathrm{sm}}} \alpha_{i} f_{i}\left(x_{0}\right)\right) \leq \mu\left(x_{0}\right),
$$

where $\alpha_{i} \geq 0, \sum_{i \in I_{\mathrm{sm}}} \alpha_{i}=1$.

Proof. If $V_{\min }$ is differentiable at $x_{0}$, then there is an integer $k$ such that $V_{\min }\left(x_{0}\right)=V_{k}\left(x_{0}\right)<$ $V_{s}\left(x_{0}\right)$ for all $s \neq k$, and $\dot{V}_{\min }\left(x_{0} ; f_{i}\left(x_{0}\right)\right)=\dot{V}_{k}\left(x_{0} ; f_{i}\left(x_{0}\right)\right)$. One has

$$
\begin{aligned}
\dot{V}_{k}\left(x_{0} ; f_{i}\left(x_{0}\right)\right) & =\sum_{i=1}^{N_{i}} \lambda_{i j} \dot{V}_{k}\left(x_{0} ; f_{i j}\left(x_{0}\right)\right) \leq \max _{j \in\left[1, N_{i}\right]}\left\{\dot{V}_{k}\left(x_{0} ; f_{i j}\left(x_{0}\right)\right)\right\} \\
& =\min _{i \in[1, N]}\left\{\max _{j \in\left[1, N_{i}\right]}\left\{\dot{V}_{k}\left(x_{0} ; f_{i j}\left(x_{0}\right)\right)\right\}\right\}=\mu\left(x_{0}\right), \quad \forall i \in \sigma\left(x_{0}\right), \forall s \notin \sigma\left(x_{0}\right) .
\end{aligned}
$$

For the structure of the switching rule (3.1), we have

$$
\max _{j \in I\left[1, N_{i}\right]}\left\{\dot{V}_{k}\left(x_{0} ; f_{i j}\left(x_{0}\right)\right)\right\}<\max _{j \in I\left[1, N_{s}\right]}\left\{\dot{V}_{k}\left(x_{0} ; f_{s j}\left(x_{0}\right)\right)\right\}, \quad \forall i \in \sigma\left(x_{0}\right), s \notin \sigma\left(x_{0}\right) .
$$

Since $V_{k}\left(x ; f_{i}(x)\right)$ is continuous in $x_{0}, \max _{j \in\left[1, N_{i}\right]}\left\{V_{k}\left(x ; f_{i j}(x)\right)\right\}$ is continuous in $x_{0}$ too. There is a small neighborhood of $x_{0}$, say $U\left(x_{0}\right)$, where

$$
\max _{j \in I\left[1, N_{i}\right]}\left\{\dot{V}_{k}\left(x ; f_{i j}(x)\right)\right\}<\max _{j \in I\left[1, N_{s}\right]}\left\{\dot{V}_{k}\left(x ; f_{s j}(x)\right)\right\}, \quad \forall i \in \sigma\left(x_{0}\right), s \notin \sigma\left(x_{0}\right),
$$

which implies $\sigma(x) \subset \sigma\left(x_{0}\right)$. We can conclude that if $s \notin \sigma\left(x_{0}\right), f_{s}(x)$ will not be chosen by the switching rule for $x \in U\left(x_{0}\right)$, that is $I_{\mathrm{sm}} \subset \sigma\left(x_{0}\right)$, and (3.5) comes from (3.7).

Moreover, along the sliding direction $\sum_{i \in I_{\mathrm{sm}}} \alpha_{i} f_{i}\left(x_{0}\right)$ we have

$$
\begin{aligned}
\dot{V}_{\min }\left(x_{0} ; \sum_{i \in I_{\mathrm{sm}}} \alpha_{i} f_{i}\left(x_{0}\right)\right) & =\left(\nabla V_{k}\left(x_{0}\right)\right)^{T} \cdot \sum_{i \in I_{\mathrm{sm}}} \alpha_{i}\left(\sum_{j=1}^{N_{i}} \lambda_{i j} f_{i j}\left(x_{0}\right)\right) \\
& =\sum_{i \in I_{\mathrm{sm}}} \alpha_{i} \sum_{j=1}^{N_{i}} \lambda_{i j}\left(\nabla V_{k}\left(x_{0}\right)\right)^{T} f_{i j}\left(x_{0}\right) \\
& =\sum_{i \in I_{\mathrm{sm}}} \alpha_{i} \sum_{j=1}^{N_{i}} \lambda_{i j} \dot{V}_{k}\left(x_{0} ; f_{i j}\left(x_{0}\right)\right) \\
& \leq \sum_{i \in I_{\mathrm{sm}}} \alpha_{i} \mu\left(x_{0}\right) \\
& =\mu\left(x_{0}\right) .
\end{aligned}
$$

Now we consider the case that $V_{\min }$ is not differentiable at $x_{0}$; that is, $J_{\min }\left(x_{0}\right)$ has two or more integers. Suppose that $J_{\min }\left(x_{0}\right)=I[1, k]$, then $V_{1}\left(x_{0}\right)=V_{2}\left(x_{0}\right)=\cdots=V_{k}\left(x_{0}\right)<V_{s}\left(x_{0}\right)$ 
for all $s>k$. When $k=2$, then $V_{1}\left(x_{0}\right)=V_{2}\left(x_{0}\right)<V_{j}\left(x_{0}\right)$, for all $j>0$. Furthermore, there must exist two $f_{i}^{\prime}$ s, say, $f_{i_{1}}$, and $f_{i_{2}}$. In this situation $I_{\mathrm{sm}}=\left\{i_{1}, i_{2}\right\}$.

Defining

$$
\Phi_{j}=\left\{x \in R^{n} \mid V_{j}(x)<V_{k}(x), \forall k \neq j\right\},
$$

and within the vicinity of $x_{0}$, we have two cases.

(a) If $x \in \Phi_{1}$, then $i_{1} \in \sigma(x)$ and a trajectory of $\dot{x}=f_{i_{1}}(x)$ would enter $\Phi_{2}$ from $\Phi_{1}$.

(b) If $x \in \Phi_{2}$, then $i_{2} \in \sigma(x)$ and a trajectory of $\dot{x}=f_{i_{2}}(x)$ would enter $\Phi_{1}$ from $\Phi_{2}$.

We interpreter cases (a) and (b) below. Case (a) implies that $f_{i_{1}}(x)$ is chosen by the switching law in $\Phi_{1}$, thus one has

$$
\max _{j \in I\left[1, N_{i_{1}}\right]}\left\{\dot{V}_{1}\left(x ; f_{i_{1} j}(x)\right)\right\} \leq \max _{j \in I\left[1, N_{s}\right]}\left\{\dot{V}_{1}\left(x ; f_{s j}(x)\right)\right\}
$$

for all $s \in I[1, N]$. In particular, one has

$$
\max _{j \in I\left[1, N_{i_{1}}\right]}\left\{\dot{V}_{1}\left(x ; f_{i_{1} j}(x)\right)\right\} \leq \max _{j \in I\left[1, N_{i_{2}}\right]}\left\{\dot{V}_{1}\left(x ; f_{i_{2} j}(x)\right)\right\}
$$

Taking $x \rightarrow x_{0}$, then

$$
\max _{j \in I\left[1, N_{i_{1}}\right]}\left\{\dot{V}_{1}\left(x_{0} ; f_{i_{1} j}\left(x_{0}\right)\right)\right\} \leq \max _{j \in I\left[1, N_{s}\right]}\left\{\dot{V}_{1}\left(x_{0} ; f_{s j}\left(x_{0}\right)\right)\right\}, \quad \forall s .
$$

When $s=i_{2}$, we have

$$
\max _{j \in I\left[1, N_{i_{1}}\right]}\left\{\dot{V}_{1}\left(x_{0} ; f_{i_{1} j}\left(x_{0}\right)\right)\right\} \leq \max _{j \in I\left[1, N_{i_{2}}\right]}\left\{\dot{V}_{1}\left(x_{0} ; f_{i_{2} j}\left(x_{0}\right)\right)\right\} .
$$

To ensure that a trajectory of $\dot{x}=f_{i_{1}}(x)$ would enter $\Phi_{2}$ from $\Phi_{1}$, we must have

$$
\max _{j \in I\left[1, N_{i_{1}}\right]}\left\{\dot{V}_{2}\left(x_{0} ; f_{i_{1} j}\left(x_{0}\right)\right)\right\} \leq \max _{j \in I\left[1, N_{i_{1}}\right]}\left\{\dot{V}_{1}\left(x_{0} ; f_{i_{1} j}\left(x_{0}\right)\right)\right\}
$$

Similarly, from case (b), we have

$$
\begin{gathered}
\max _{j \in I\left[1, N_{i_{2}}\right]}\left\{\dot{V}_{2}\left(x_{0} ; f_{i_{2} j}\left(x_{0}\right)\right)\right\} \leq \max _{j \in I\left[1, N_{s}\right]}\left\{\dot{V}_{2}\left(x_{0} ; f_{s j}\left(x_{0}\right)\right)\right\}, \quad \forall s, \\
\max _{j \in I\left[1, N_{i_{2}}\right]}\left\{\dot{V}_{2}\left(x_{0} ; f_{i_{2} j}\left(x_{0}\right)\right)\right\} \leq \max _{j \in I\left[1, N_{i_{1}}\right]}\left\{\dot{V}_{2}\left(x_{0} ; f_{i_{1} j}\left(x_{0}\right)\right)\right\}, \\
\max _{j \in I\left[1, N_{i_{2}}\right]}\left\{\dot{V}_{1}\left(x_{0} ; f_{i_{2} j}\left(x_{0}\right)\right)\right\} \leq \max _{j \in I\left[1, N_{i_{2}}\right]}\left\{\dot{V}_{2}\left(x_{0} ; f_{i_{2} j}\left(x_{0}\right)\right)\right\} .
\end{gathered}
$$


Combining the inequalities from (3.15) to (3.19), one has

$$
\begin{aligned}
\max _{j \in I\left[1, N_{i_{1}}\right]}\left\{\dot{V}_{1}\left(x_{0} ; f_{i_{1} j}\left(x_{0}\right)\right)\right\} & \leq \max _{j \in I\left[1, N_{i_{2}}\right]}\left\{\dot{V}_{1}\left(x_{0} ; f_{i_{2} j}\left(x_{0}\right)\right)\right\} \\
& \leq \max _{j \in I\left[1, N_{i_{2}}\right]}\left\{\dot{V}_{2}\left(x_{0} ; f_{i_{2} j}\left(x_{0}\right)\right)\right\} \\
& \leq \max _{j \in I\left[1, N_{i_{1}}\right]}\left\{\dot{V}_{2}\left(x_{0} ; f_{i_{1} j}\left(x_{0}\right)\right)\right\} \\
& \leq \max _{j \in I\left[1, N_{i_{1}}\right]}\left\{\dot{V}_{1}\left(x_{0} ; f_{i_{1} j}\left(x_{0}\right)\right)\right\} .
\end{aligned}
$$

Thus, the following inequalities:

$$
\begin{aligned}
\max _{j \in I\left[1, N_{i_{1}}\right]}\left\{\dot{V}_{1}\left(x_{0} ; f_{i_{1} j}\left(x_{0}\right)\right)\right\} & =\max _{j \in I\left[1, N_{i_{2}}\right]}\left\{\dot{V}_{1}\left(x_{0} ; f_{i_{2} j}\left(x_{0}\right)\right)\right\} \\
& =\max _{j \in I\left[1, N_{i_{2}}\right]}\left\{\dot{V}_{2}\left(x_{0} ; f_{i_{2} j}\left(x_{0}\right)\right)\right\} \\
& =\max _{j \in I\left[1, N_{i_{1}}\right]}\left\{\dot{V}_{2}\left(x_{0} ; f_{i_{1} j}\left(x_{0}\right)\right)\right\},
\end{aligned}
$$

are valid. They are all equal to $\mu\left(x_{0}\right)$. So we have

$$
\begin{aligned}
\dot{V}_{\min }\left(x_{0} ; f_{i_{1}}\left(x_{0}\right)\right) & =\min \left\{\dot{V}_{1}\left(x_{0} ; f_{i_{1}}\left(x_{0}\right)\right), \dot{V}_{2}\left(x_{0} ; f_{i_{1}}\left(x_{0}\right)\right)\right\} \\
& =\min \left\{\dot{V}_{1}\left(x_{0} ; \sum_{j=1}^{N_{i_{1}}} \lambda_{i_{1} j} f_{i_{1} j}\left(x_{0}\right)\right), \dot{V}_{2}\left(x_{0} ; \sum_{j=1}^{N_{i_{1}}} \lambda_{i_{1} j} f_{i_{1} j}\left(x_{0}\right)\right)\right\} \\
& \leq \min \left\{\max _{j \in I\left[1, N_{i_{1}}\right]}\left\{\dot{V}_{1}\left(x_{0} ; f_{i_{1} j}\left(x_{0}\right)\right)\right\}, \max _{j \in I\left[1, N_{i_{1}}\right]}\left\{\dot{V}_{2}\left(x_{0} ; f_{i_{1} j}\left(x_{0}\right)\right)\right\}\right\} \\
& =\mu\left(x_{0}\right) .
\end{aligned}
$$

Similarly, we obtain $\dot{V}_{\min }\left(x_{0} ; f_{i_{2}}\left(x_{0}\right)\right) \leq \mu\left(x_{0}\right)$. Thus the relation (3.5) follows for $I_{\mathrm{sm}}=\left\{i_{1}, i_{2}\right\}$. The above argument can be extended to the case $k>2$. Then $V_{1}, V_{2}, \ldots, V_{k}$ are involved in the sliding motion with corresponding indices $i_{1}, i_{2}, \ldots, i_{k}$, with $f_{i_{1}}\left(x_{0}\right)$ pointing from $\Phi_{1}$ to $\Phi_{2}, \ldots, f_{i_{k}}\left(x_{0}\right)$ pointing from $\Phi_{k}$ to $\Phi_{1}$. Similar procedure can be used to derive the conclusion extended from (3.21).

$$
\max _{j \in I\left[1, N_{i_{s}}\right]}\left\{\dot{V}_{s}\left(x_{0} ; f_{i_{s} j}\left(x_{0}\right)\right)\right\}=\max _{j \in I\left[1, N_{i_{t}}\right]}\left\{\dot{V}_{l}\left(x_{0} ; f_{i_{j} j}\left(x_{0}\right)\right)\right\}=\mu\left(x_{0}\right), \quad \forall s, t, l \in I[1, k] .
$$


Thus we have

$$
\begin{aligned}
\dot{V}_{\min }\left(x_{0} ; f_{i_{1}}\left(x_{0}\right)\right) & =\min \left\{\dot{V}_{t}\left(x_{0} ; f_{i_{1} j}\left(x_{0}\right)\right), t \in I[1, k]\right\} \\
& =\min \left\{\sum_{j=1}^{N_{i_{1}}} \mu_{i_{1} j} \dot{V}_{t}\left(x_{0} ; f_{i_{1} j}\left(x_{0}\right)\right), t \in I[1, k]\right\} \\
& \leq \min \left\{\max _{j \in I\left[1, N_{i_{1}}\right]}\left\{\dot{V}_{t}\left(x_{0} ; f_{i_{1}}\left(x_{0}\right)\right)\right\}, t \in I[1, k]\right\} \\
& =\mu\left(x_{0}\right) .
\end{aligned}
$$

Similarly, we have $\dot{V}_{\min }\left(x_{0} ; f_{i_{2}}\left(x_{0}\right)\right) \leq \mu\left(x_{0}\right), \ldots, \dot{V}_{\min }\left(x_{0} ; f_{i_{k}}\left(x_{0}\right)\right) \leq \mu\left(x_{0}\right)$. Thus the relation (3.5) is obtained for $I_{\mathrm{sm}}=\left\{i_{1}, i_{2}, \ldots, i_{k}\right\}$.

Now we consider there are three elements in the sliding direction. Let $v_{1}, v_{2}, v_{3}$ be positive numbers, such that $v_{1}+v_{2}+v_{3}=1$. Define $\xi=\left(v_{1} f_{i_{1}}+v_{2} f_{i_{2}}+v_{3} f_{i_{3}}\right)\left(x_{0}\right)$. Since sliding mode stays in the set where $V_{1}(x)=V_{2}(x)=V_{3}(x)$, we have

$$
\begin{gathered}
\dot{V}_{1}\left(x_{0} ; \xi\right)=\dot{V}_{2}\left(x_{0} ; \xi\right)=\dot{V}_{3}\left(x_{0} ; \xi\right)=\dot{V}_{\min }\left(x_{0} ; \xi\right), \\
V_{1}\left(x_{0}\right)=V_{2}\left(x_{0}\right)=V_{3}\left(x_{0}\right)=V_{\min }\left(x_{0}\right) .
\end{gathered}
$$

For $\Delta t>0$, let $x_{1}=x_{0}+\Delta t v_{1} f_{i_{1}}\left(x_{0}\right), x_{2}=x_{0}+\Delta t\left(v_{1} f_{i_{1}}\left(x_{0}\right)+v_{2} f_{i_{2}}\left(x_{0}\right)\right)$, and $x_{3}=x_{0}+\Delta t \xi$. Due to the sliding motion for $\Delta t$ sufficient small, $x_{1} \in \Phi_{2}, x_{2} \in \Phi_{3}$ and by (3.25), $V_{j}\left(x_{3}\right)-V_{\min }\left(x_{3}\right)=$ $o(\Delta t), j=1,2,3$ :

$$
\begin{aligned}
\dot{V}_{\min }\left(x_{0} ; \xi\right)= & \lim _{\Delta t \downarrow 0} \frac{V_{\min }\left(x_{0}+\Delta t \xi\right)-V_{\min }\left(x_{0}\right)}{\Delta t}=\lim _{\Delta t \downarrow 0} \frac{V_{\min }\left(x_{3}\right)-V_{\min }\left(x_{0}\right)}{\Delta t} \\
= & \lim _{\Delta t \downarrow 0} \frac{V_{\min }\left(x_{3}\right)-V_{\min }\left(x_{2}\right)}{\Delta t}+\lim _{\Delta t \downarrow 0} \frac{V_{\min }\left(x_{2}\right)-V_{\min }\left(x_{1}\right)}{\Delta t} \\
& +\lim _{\Delta t \downarrow 0} \frac{V_{\min }\left(x_{1}\right)-V_{\min }\left(x_{0}\right)}{\Delta t} \\
\leq & \lim _{\Delta t \downarrow 0} \frac{V_{3}\left(x_{3}\right)-V_{3}\left(x_{2}\right)}{\Delta t}+\lim _{\Delta t \downarrow 0} \frac{V_{2}\left(x_{2}\right)-V_{2}\left(x_{1}\right)}{\Delta t} \\
& +\lim _{\Delta t \downarrow 0} \frac{V_{1}\left(x_{1}\right)-V_{1}\left(x_{0}\right)}{\Delta t} .
\end{aligned}
$$


Since each function $V_{j}, j=1,2,3$ is continuously differentiable, then

$$
\begin{aligned}
\dot{V}_{\min }( & \left.x_{0} ;\left(v_{1} f_{i_{1}}\left(x_{0}\right)+v_{2} f_{i_{2}}\left(x_{0}\right)+v_{3} f_{i_{3}}\left(x_{0}\right)\right)\right) \\
= & \dot{V}_{\min }\left(x_{0} ; \xi\right) \\
\leq & v_{1} \dot{V}_{1}\left(x_{0} ; f_{i_{1}}\left(x_{0}\right)\right)+v_{2} \dot{V}_{2}\left(x_{0} ; f_{i_{2}}\left(x_{0}\right)\right)+v_{3} \dot{V}_{3}\left(x_{0} ; f_{i_{3}}\left(x_{0}\right)\right) \\
= & v_{1} \dot{V}_{1}\left(x_{0} ; \sum_{j=1}^{N_{i_{1}}} v_{i_{1} j} f_{i_{1} j}\left(x_{0}\right)\right)+v_{2} \dot{V}_{2}\left(x_{0} ; \sum_{j=1}^{N_{i_{2}}} v_{i_{2} j} f_{i_{2} j}\left(x_{0}\right)\right) \\
& +v_{3} \dot{V}_{3}\left(x_{0} ; \sum_{j=1}^{N_{i_{3}}} v_{i_{3} j} f_{i_{3} j}\left(x_{0}\right)\right) \\
\leq & v_{1} \max _{i \in I\left[1, N_{i_{1}}\right]}\left\{\dot{V}_{1}\left(x_{0} ; f_{i_{1} j}\left(x_{0}\right)\right)\right\}+v_{2} \max _{i \in I\left[1, N_{i_{2}}\right]}\left\{\dot{V}_{2}\left(x_{0} ; f_{i_{2} j}\left(x_{0}\right)\right)\right\} \\
& +v_{3} \max _{j \in I\left[1, N_{i_{3}}\right]}\left\{\dot{V}_{3}\left(x_{0} ; f_{i_{3} j}\left(x_{0}\right)\right)\right\}=\mu\left(x_{0}\right) .
\end{aligned}
$$

Above step can be extended to the case where $I_{\mathrm{sm}}$ has more than three elements. Therefore, the inequality (3.6) is satisfied and the proof is completed.

To summarize Propositions 3.1 and 3.2 we have the result that if $x$ is not in a sliding mode, then $\dot{x}=f_{\sigma(x)}(x)$ and $\dot{V}_{\min }(x ; \dot{x})=\dot{V}_{\min }\left(x ; f_{\sigma(x)}(x)\right) \leq \mu(x)$. If $x$ is in a sliding mode involving subsystems $\dot{x}=f_{i}(x), i \in I_{\mathrm{sm}}$, then there exist $\alpha_{i}, i \in I_{\mathrm{sm}}$, satisfying $0 \leq \alpha_{i} \leq 1$, and $\sum_{i \in I_{\mathrm{sm}}} \alpha_{i}=1$, such that $\dot{x}=\sum_{i \in I_{\mathrm{sm}}} \alpha_{i} f_{i}(x)$. We also have $\dot{V}_{\min }(x ; \dot{x}) \leq \mu(x)$ from (3.6). In view of these arguments, we can establish a sufficient condition of stability for the switched systems in terms of $\mu(x)$.

Theorem 3.3. For the switched system (2.1) under the switching rule (3.1), if there exists an $\eta \in R$, such that

$$
\min _{i \in I[1, N]}\left\{\max _{j \in I\left[1, N_{i}\right]} \dot{V}_{\min }\left(x ; f_{i j}(x)\right)\right\}=\mu(x) \leq \eta V_{\min }(x)
$$

for all $x \in R^{n} \backslash\{0\}$, then

$$
V_{\min }(x(t)) \leq V_{\min }(x(0)) e^{\eta t}
$$

for every solution $x(\cdot)$.

Remark 3.4. This theorem indicates that when a minimum function is used, it is sufficient to use the directional derivatives along all vertex directions of subsystems to characterize stability of the switched systems with polytopic uncertainties, and the existence of sliding modes has no effect. 
When we consider the the case of the switched linear systems, then the systems (2.1) turn to

$$
\begin{gathered}
\dot{x}(t)=A_{\sigma(x, t)} x(t), \\
A_{i}=\sum_{j=1}^{N_{i}} \lambda_{i j} A_{i j}, \quad i \in I[1, N],
\end{gathered}
$$

where $A_{i j}, j \in I\left[1, N_{i}\right], i \in I[1, N]$ are constant matrices.

We can establish a matrix condition for exponential stabilizability of the systems.

Theorem 3.5. The switched linear system (3.30) is exponentially stabilizable via state switching if there exist real matrices $P_{j}=P_{j}^{T}>0, j \in I[1, J]$, and real number $\eta, 0 \leq \mu_{i s_{i} j} \leq 1, \sum_{i=1}^{N} \mu_{i s_{i} j}=1$, $\beta_{j k} \geq 0, j, k \in I[1, J], s_{i} \in I\left[1, N_{i}\right], i \in I[1, N]$, such that

$$
\left(\sum_{i=1}^{N} \mu_{i s_{i} j} A_{i s_{i}}\right)^{T} P_{j}+P_{j}\left(\sum_{i=1}^{N} \mu_{i s_{i} j} A_{i s_{i}}\right) \leq \sum_{k=1}^{J} \beta_{j k}\left(P_{j}-P_{k}\right)+\eta P_{j} .
$$

Proof. Let $V_{\min }(x)=\min \left\{x^{T} P_{j} x \mid j \in I[1, J]\right\}$. Consider $x \neq 0$ and assume that $J_{\min }(x)=$ $\left\{1,2, \ldots, J_{0}\right\}$ for a certain integer $J_{0} \leq J$. Then $x^{T} P_{k} x=V_{\min }(x)$ for $k \leq J_{0}$ and $x^{T} P_{k} x>V_{\min }(x)$ for $k>J_{0}$. Hence $x^{T}\left(P_{j}-P_{k}\right) x \leq 0$, for all $j \leq J_{0}$. According to (3.32), the following inequalities:

$$
\begin{aligned}
2 x^{T} P_{j}\left(\sum_{i=1}^{N} \mu_{i s_{i} j} A_{i s_{i}}\right) x & \leq \sum_{k=1}^{J} \beta_{j k} x^{T}\left(P_{j}-P_{k}\right) x+\eta x^{T} P_{j} x \\
& \leq \eta x^{T} P_{j} x=\eta V_{\min }(x), \quad \forall j \leq J_{0},
\end{aligned}
$$

hold.

Since $0 \leq \mu_{i s_{i} j} \leq 1$, we have

$$
\min \left\{2 x^{T} P_{j} A_{i s_{i}} x: s_{i} \in I\left[1, N_{i}\right], i \in I[1, N]\right\} \leq \eta V_{\min }(x), \quad \forall j \leq J_{0}
$$

It follows that

$$
\min _{i \in I[1, N]}\left\{\max _{s_{i} \in I\left[1, N_{i}\right]}\left\{2 x^{T} P_{j} A_{i s_{i}} x\right\}\right\} \leq \eta V_{\min }(x), \quad \forall j \leq J_{0} .
$$


By Lemma A.1 in the appendix,

$$
\begin{aligned}
\min _{i \in I[1, N]}\left\{\max _{s \in I\left[1, N_{i}\right]} \dot{V}_{\min }\left(x ; A_{i s_{i}} x\right)\right\} & =\min _{i \in I[1, N]}\left\{\max _{s_{i} \in I\left[1, N_{i}\right]} \min \left\{\dot{V}_{j}\left(x ; A_{i s_{i}} x\right), j \in J_{\min }(x)\right\}\right\} \\
& =\min _{i \in I[1, N]}\left\{\max _{s_{i} \in I\left[1, N_{i}\right]} \min \left\{2 x^{T} P_{j} A_{i s_{i}} x, j \in J_{\min }(x)\right\}\right\} \\
& \leq \min _{i \in I[1, N]}\left\{\max _{s_{i} \in I\left[1, N_{i}\right]}\left\{2 x^{T} P_{1} A_{i s_{i}} x\right\}\right\} \leq \eta V_{\min }(x) .
\end{aligned}
$$

Define the switching rule as

$$
\sigma(x)=\underset{i \in I[1, N]}{\arg \min }\left\{\max _{j \in I\left[1, N_{i}\right]} \dot{V}_{\min }\left(x ; A_{i j} x\right)\right\} .
$$

Under this switching rule we have $V_{\min }(x(t)) \leq V_{\min }(x(0)) e^{\eta t}$ for every solution $x(\cdot)$ from Theorem 3.3. The proof is completed.

Remark 3.6. If $P_{j}=P$ for all $j \in I[1, J]$ and $N=2$, then $V_{\min }$ can be reduced to a quadratic function and (3.32) can be reduced to the matrix inequalities

$$
\left[\mu_{s t} A_{1 s}+\left(1-\mu_{s t}\right) A_{2 t}\right]^{T} P+P\left[\mu_{s t} A_{1 s}+\left(1-\mu_{s t}\right) A_{2 t}\right]<\eta_{0} P .
$$

If $\eta_{0}=0$, then this corresponds to the stability condition given in [14]. In this sense, Theorem 3.5 is the extension of the main result in [14].

Example 3.7. Consider the switched linear system (3.30) composed of two subsystems, where

$$
\begin{array}{cc}
A_{11}=\left[\begin{array}{cc}
2 & 1 \\
2 & -4
\end{array}\right], & A_{12}=\left[\begin{array}{cc}
2 & 1 \\
1 & -6
\end{array}\right], \\
A_{21}=\left[\begin{array}{cc}
-4 & -1 \\
0 & 2.1
\end{array}\right], & A_{22}=\left[\begin{array}{cc}
-5 & -1 \\
0.1 & 2
\end{array}\right] .
\end{array}
$$

The eigenvalues of $A_{11}, A_{12}, A_{21}, A_{22}$ are $\{2.3166,-4.3166\},\{-6.1231,2.1231\},\{-4,2.1\}$, and $\{-4.9857,1.9857\}$, respectively. They are all unstable. Therefore, neither subsystems is quadratically stable.

We turn to use $V_{\min }$ composed of two quadratic functions and minimize $\eta$ subject to (3.32). The minimal $\eta$ is given as -1.4752 when fix the parameters as following: $\mu_{11}=0.5$, $\mu_{12}=0.5, \mu_{21}=0.4, \mu_{22}=0.6$. That is to say a unified convergence rate is guaranteed. For 


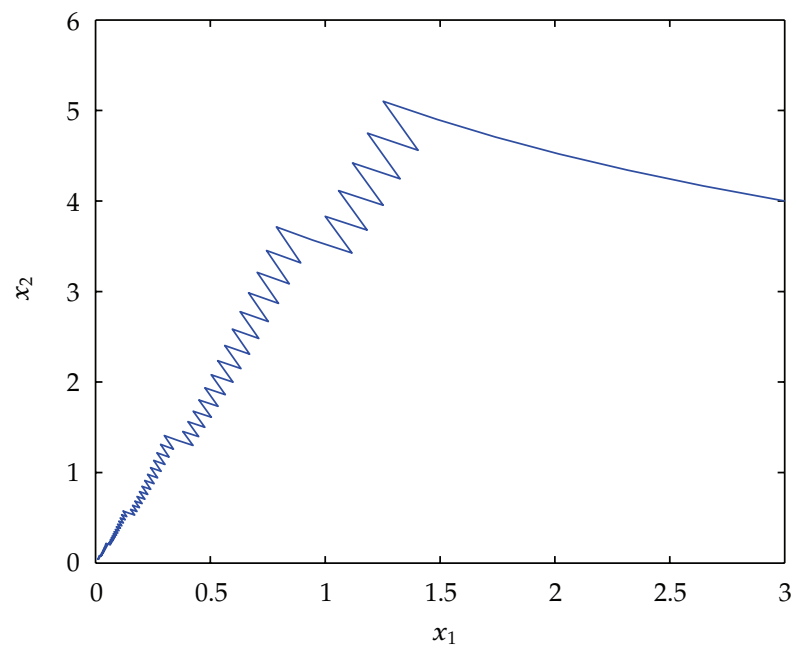

Figure 1: The state of the switched system in example.

verification, other parameters are provided as following:

$$
\begin{aligned}
P_{1}=\left[\begin{array}{cc}
11.5608 & 0.4678 \\
0.4678 & 2.1290
\end{array}\right], \quad P_{2}=\left[\begin{array}{cc}
11.5591 & 0.4723 \\
0.4723 & 2.1177
\end{array}\right], \\
\beta_{12}=8.9, \quad \beta_{21}=5.8 .
\end{aligned}
$$

Let us investigate the system state trajectory using the two special subsystems

$$
\begin{aligned}
& A_{1}=0.4 A_{11}+0.6 A_{12}=\left[\begin{array}{cc}
2 & 1 \\
1.4 & -5.2
\end{array}\right], \\
& A_{2}=0.1 A_{21}+0.9 A_{22}=\left[\begin{array}{cc}
-4.9 & -1 \\
0.09 & 2.01
\end{array}\right] .
\end{aligned}
$$

They are both unstable.

We suppose that the initial state is $x_{0}=[3,4]^{T}$. According to the switched rule (3.37), the dynamic system can exponential stabilizability and the typical result is plotted in Figure 1, which show that the system state converge to zero very quickly.

\section{Conclusion}

In this paper, the exponential stabilizability of switched nonlinear systems with polytopic uncertainties is considered employing the methods of nonsmooth analysis and nonquadratic Lyapunov functions. The function is formed by taking the pointwise minimum of a family of quadratic functions. We establish the switching rule to stabilize the switched systems by utilizing the directional derivative along the vertex directions of subsystems. In this process, we take a lot of effort in examining the case that the control systems involving sliding modes. The matrix conditions for exponential stabilizability of the switched linear systems are also 
obtained. As numerical example demonstrate in this paper, our synthesis result is effective. Future efforts will be devoted to the switched systems with time delay.

\section{Appendix}

For convenience we briefly list some definitions and conclusions from nonsmooth analysis. One can refer to $[29,30]$ for more detail.

Suppose $f$ is defined from $R^{n}$ to $\bar{R}=R \bigcup\{-\infty,+\infty\}$, and $f(x)$ is finite. The one-sided directional derivative of $f$ at $x$ in direction $\zeta$ can be expressed by

$$
\dot{f}(x ; \zeta)=\lim _{\Delta t \downarrow 0} \frac{f(x+\zeta \Delta t)-f(x)}{\Delta t} .
$$

Suppose $f$ is a convex function in $R^{n}$, and finite at $x$. The set

$$
\partial f(x)=\left\{x^{*} \mid f(z) \geq f(x)+x^{* T}(z-x), \forall z \in R^{n}\right\}
$$

is called the subdifferential of $f$ at $x$ and $x^{*}$ is a subgradient of $f$ at $x$ [30]. For a convex function $f$ in $R^{n}, f$ is differential at $x_{0}$ if and only if $\partial f\left(x_{0}\right)$ has only one vector. In this case we have $\partial f\left(x_{0}\right)=\nabla f\left(x_{0}\right)$.

If $f$ is locally Lipschitz near $x$ and $S$ is any set of Lebesgue measure 0 in $R^{n}$. The set of points at which $f$ fails to be differentiable is denoted by $\Omega_{f}$. Then the generalized gradient of $f$ at $x$ in the sense of Clarke denoted by $\partial_{C} f(x)$ is defined as

$$
\partial_{C} f(x)=\operatorname{co}\left\{\lim _{x_{i} \rightarrow x} \nabla f\left(x_{i}\right) \mid x_{i} \notin S, x_{i} \notin \Omega_{f}\right\}
$$

The following lemma can be obtained by combining some results from $[29,31]$.

Lemma A.1. Suppose $x_{0} \in R^{n}$. Then one has

(1) For $\zeta \in R^{n}$, the directional derivative of $V_{\min }$ at $x_{0}$ along $\zeta$ is given by

$$
\dot{V}_{\min }\left(x_{0} ; \zeta\right)=\min \left\{\dot{V}_{j}\left(x_{0} ; \zeta\right) \mid j \in J_{\min }\left(x_{0}\right)\right\}
$$

where the index set $J_{\min }\left(x_{0}\right)=\left\{j \in I[1, J] \mid V_{j}\left(x_{0}\right)=V_{\min }\left(x_{0}\right)\right\}$.

(2) If $V_{j}(x)=x^{T} P_{j} x, j \in I[1, J]$, then $\partial_{C} V_{\min }\left(x_{0}\right)=\operatorname{co}\left\{2 P_{j} x_{0} \mid j \in J_{\min }\left(x_{0}\right)\right\}$.

\section{Acknowledgment}

This work was supported by the National Natural Science Foundation of China (under Grant: 10971187, 11071029, and 11201362). 


\section{References}

[1] W. P. Dayawansa and C. F. Martin, "A converse Lyapunov theorem for a class of dynamical systems which undergo switching," IEEE Transactions on Automatic Control, vol. 44, no. 4, pp. 751-760, 1999.

[2] H. Lin and P. J. Antsaklis, "Stability and stabilizability of switched linear systems: a survey of recent results," IEEE Transactions on Automatic Control, vol. 54, no. 2, pp. 308-322, 2009.

[3] R. W. Brockett, "Asymptotic stability and feedback stabilization," in Differential Geometric Control Theory, vol. 27, pp. 181-191, Birkhäuser, Boston, Mass, USA, 1983.

[4] T. Hu, L. Ma, and Z. Lin, "Stabilization of switched systems via composite quadratic functions," IEEE Transactions on Automatic Control, vol. 53, no. 11, pp. 2571-2585, 2008.

[5] M. C. F. Donkers, W. P. M. H. Heemels, N. van de Wouw, and L. Hetel, "Stability analysis of networked control systems using a switched linear systems approach," IEEE Transactions on Automatic Control, vol. 56, no. 9, pp. 2101-2115, 2011.

[6] L. Gurvits, R. Shorten, and O. Mason, "On the stability of switched positive linear systems," IEEE Transactions on Automatic Control, vol. 52, no. 6, pp. 1099-1103, 2007.

[7] J. Lian and K. Zhang, "Exponential stability for switched Cohen-Grossberg neural networks with average dwell time," Nonlinear Dynamics, vol. 63, no. 3, pp. 331-343, 2011.

[8] D. Wang, W. Wang, and P. Shi, "Delay-dependent model reduction for continuous-time switched state-delayed systems," International Journal of Adaptive Control and Signal Processing, vol. 25, no. 9, pp. 843-854, 2011.

[9] W. Zhang, A. Abate, J. Hu, and M. P. Vitus, “Exponential stabilization of discrete-time switched linear systems," Automatica, vol. 45, no. 11, pp. 2526-2536, 2009.

[10] D. Liberzon, Switching in Systems and Control, Birkhäuser, Boston, Mass, USA, 2003.

[11] Z. D. Sun and S. S. Ge, Switched Linear Systems Control and Design, Springer, Heidelberg, Germany, 2004.

[12] D. Xie and G. Xu, "Computation of performance robustness bounds for control sys-tems with parameter uncertainties: an LMI approach," IEE Proceedings Control Theory E Applications, vol. 152, no. 6, pp. 675-682, 2005.

[13] N. Chen, W. H. Gui, and B. Y. Liu, "Parametric absolute stability of interconnected Lurie control systems," Acta Automatica Sinica, vol. 33, no. 12, pp. 1283-1289, 2007.

[14] G. Zhai, H. Lin, and P. J. Antsaklis, "Quadratic stabilizability of switched linear systems with polytopic uncertainties," International Journal of Control, vol. 76, no. 7, pp. 747-753, 2003.

[15] H. Lin and P. J. Antsaklis, "Switching stabilizability for continuous-time uncertain switched linear systems," IEEE Transactions on Automatic Control, vol. 52, no. 4, pp. 633-646, 2007.

[16] N. Otsuka and T. Soga, "Quadratic stablizability for polytopic uncertain continuous-time switched linear systems composed of two subsystems," International Journal of Control and Automation, vol. 3, no. 1, pp. 35-42, 2010.

[17] T. Saga and N. Otsuka, "Quadratic stabilizability for polytopic uncertain continuous-time switched linear systems via switched observer," in Proceedings of the 19th Mediterranean Conference on Control and Automation Aquis Corfu Holiday Palace, pp. 724-729, Corfu, Greece, June 2011.

[18] Z. Chen and Y. Gao, "On common linear copositive Lyapunov functions for pairs of stable positive linear systems," Nonlinear Analysis. Hybrid Systems, vol. 3, no. 4, pp. 467-474, 2009.

[19] Z. Chen and Y. Gao, "Common Lyapunov functions for stable second order systems," in Proceedings of the 1st International Workshop on Intelligent Systems and Applications, pp. 1754-1756, IEEE, 2009.

[20] B. E. A. Milani, "Piecewise-affine Lyapunov functions for continuous-time linear systems with saturating controls," in Proceedings of the American Control Conference, pp. 429-434, Boston, Mass, USA, June 2004.

[21] N. Luo, M. de la Sen, and J. Rodellar, "Robust stabilization of a class of uncertain time delay systems in sliding mode," International Journal of Robust and Nonlinear Control, vol. 7, no. 1, pp. 59-74, 1997.

[22] M. da la Sen, "Quadratic stability and stabilization of switched dynamic systems with uncommensurate internal point delays," Applied Mathematics and Computation, vol. 185, no. 1, pp. 508-526, 2007.

[23] T. Hu and Z. Lin, "Composite quadratic Lyapunov functions for constrained control systems," IEEE Transactions on Automatic Control, vol. 48, no. 3, pp. 440-450, 2003.

[24] L. Lu and Z. Lin, "Design of a nonlinear anti-windup gain by using a composite quadratic Lyapunov function," IEEE Transactions on Automatic Control, vol. 56, no. 12, pp. 2997-3001, 2011.

[25] T. Hu, R. Goebel, A. R. Teel, and Z. Lin, "Conjugate Lyapunov functions for saturated linear systems," Automatica, vol. 41, no. 11, pp. 1949-1956, 2005. 
[26] X. Dong and J. Zhao, "Solvability of the output regulation problem for switched nonlinear systems," Control Theory \& Applications, IET, vol. 6, no. 8, pp. 1130-1136, 2012.

[27] J. Lian and J. Zhao, "Sliding mode control of uncertain switched delay systems via hysteresis switching strategy," International Journal of Control, vol. 8, no. 6, pp. 1171-1178, 2010.

[28] A. F. Filippov, Differential Equations With Discontinuous Right Hand Sides, vol. 18, Kluwer Academic Publishers, Norwell, Mass, USA, 1988.

[29] F. H. Clarke, Yu. S. Ledyaev, R. J. Stern, and P. R. Wolenski, Nonsmooth Analysis and Control Theory, vol. 178, Springer, New York, NY, USA, 1998.

[30] R. T. Rockafellar, Convex Analysis, Princeton University Press, Princeton, NJ, USA, 1970.

[31] S. Boyd, Lecture Notes for Convex Optimization II, http://www.stanford.edu/class/ee364b/ lectures/subgradients_slides.pdf. 


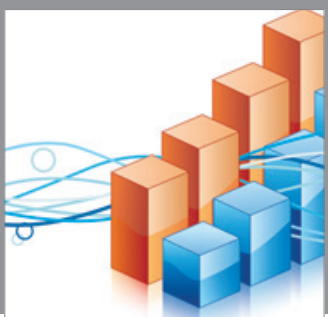

Advances in

Operations Research

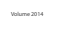

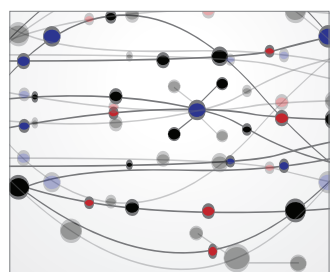

\section{The Scientific} World Journal
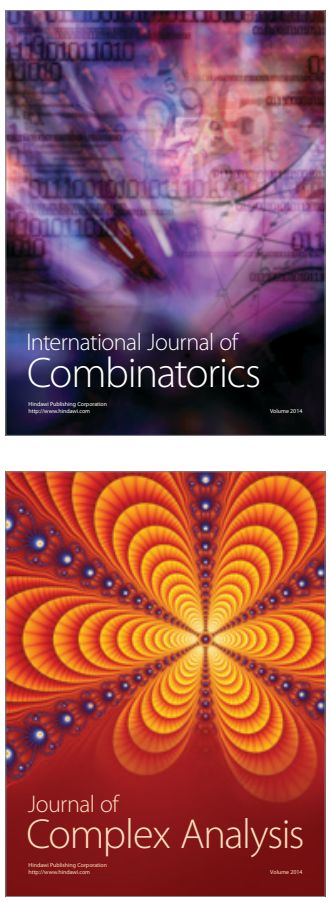

International Journal of

Mathematics and

Mathematical

Sciences
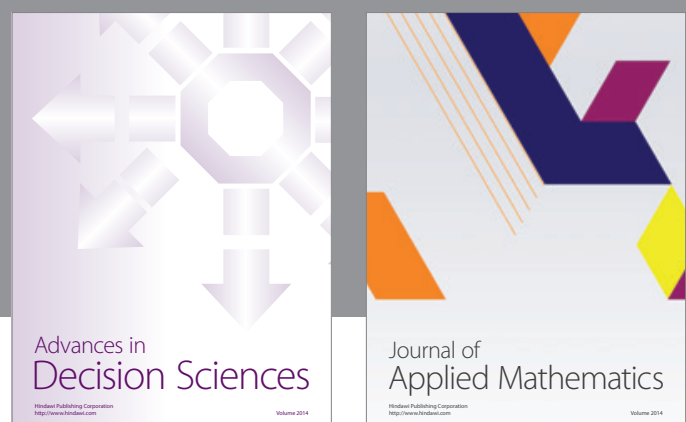

Journal of

Applied Mathematics
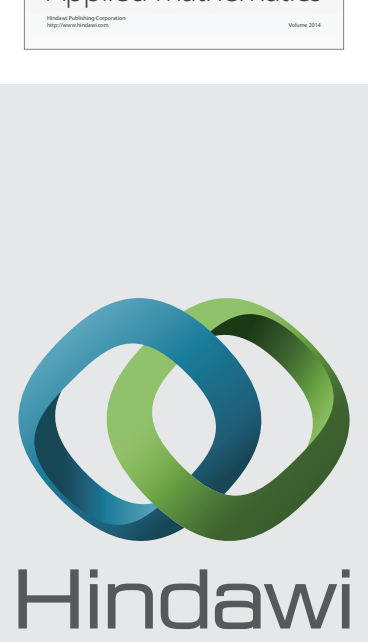

Submit your manuscripts at http://www.hindawi.com
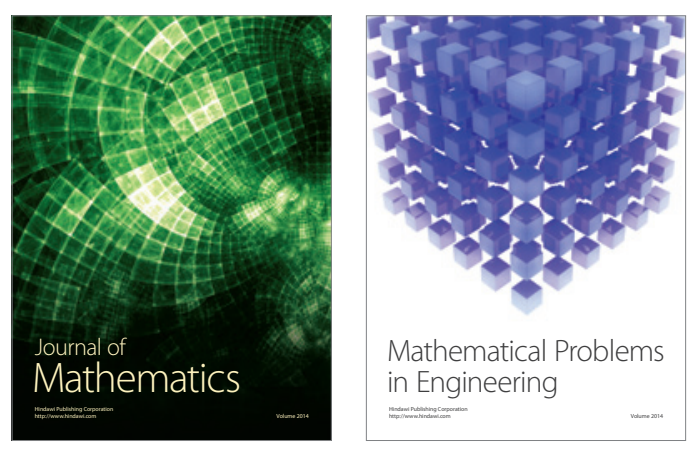

Mathematical Problems in Engineering
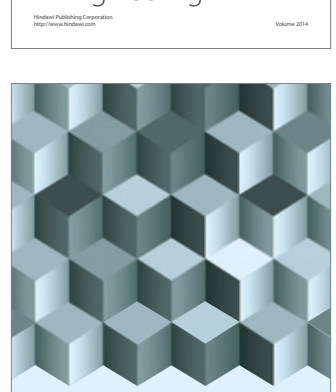

Journal of

Function Spaces
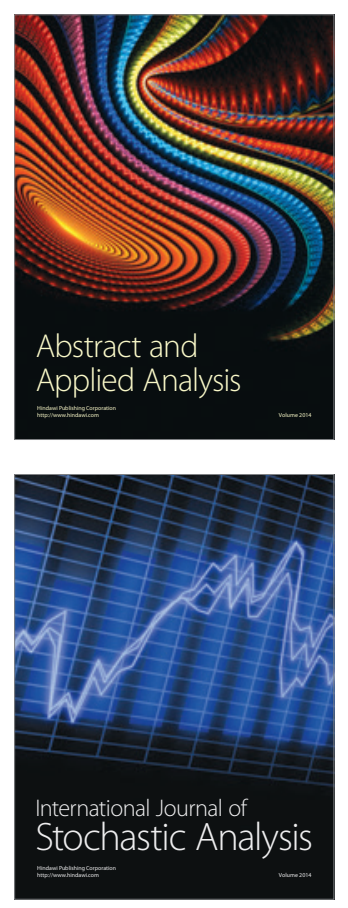

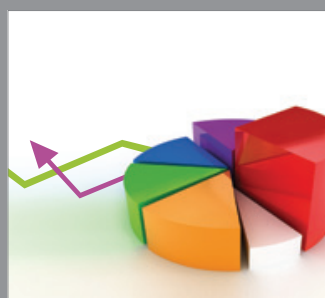

ournal of

Probability and Statistics

Promensencen
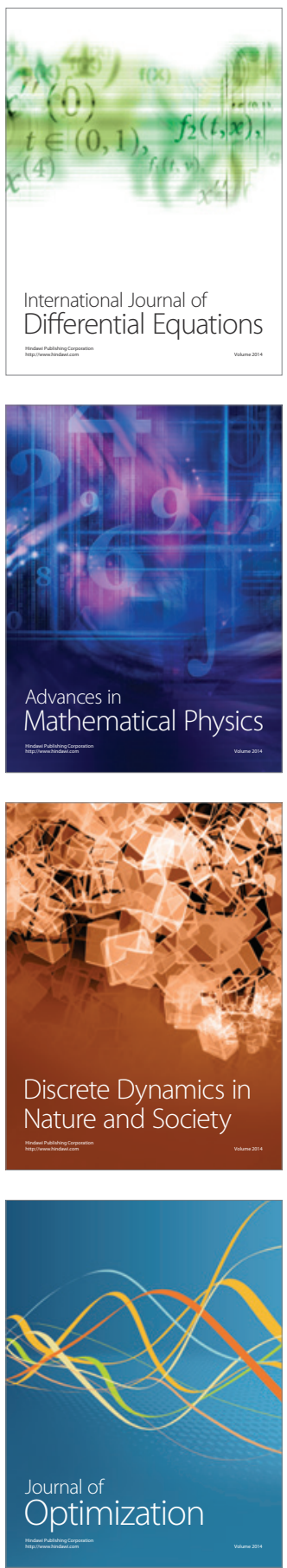